\title{
Reduction of Lateral Shoots by RNA Interference and by Chemical Mutation of Genes Involved in Axillary Meristem Regulation and Field Trials of Mutant Lines in Nicotiana Tabacum L.
}

\author{
Kaori Hamano ( $\nabla$ kaori.hamano@jt.com ) \\ Tobacco Institute Inc https://orcid.org/0000-0001-9381-6620 \\ Seiki Sato \\ Leaf Tobacco Research Center, Japan Tobacco Inc. \\ Masao Arai \\ Leaf Tobacco Research Center, Japan Tobacco Inc. \\ Yuta Negishi \\ Leaf Tobacco Research Center, Japan Tobacco Inc. \\ Takashi Nakamura \\ Leaf Tobacco Research Center, Japan Tobacco Inc. \\ Tomoyuki Komatsu \\ Leaf Tobacco Research Center, Japan Tobacco Inc. \\ Tsuyoshi Naragino \\ Leaf Tobacco Research Center, Japan Tobacco Inc. \\ Shoichi Suzuki \\ Leaf Tobacco Research Center, Japan Tobacco Inc.
}

\section{Research article}

Keywords: chemical mutation, lateral shoot reduction, RNAi, RNAseq, sucker, tobacco

Posted Date: January 6th, 2021

DOl: https://doi.org/10.21203/rs.3.rs-138960/v1

License: (c) (1) This work is licensed under a Creative Commons Attribution 4.0 International License. Read Full License 


\section{Abstract}

\section{Background}

Lateral branches vigorously proliferate in tobacco after topping of inflorescence portions of stems for maturation of leaves to be harvested. Therefore, tobacco varieties with reduced lateral shoots are highly desired by farmers.

\section{Results}

Genetic reduction of lateral shoots was attempted in tobacco. Two groups of genes were examined by RNA interference: homologues of the genes reported as involved in the formation of lateral shoots in other plants, and genes highly expressed in primordial stages of axillary buds. Although "primary" lateral shoots that grew after the plants were topped off when flower buds emerged were not much affected, "secondary" lateral shoots, which appeared from the abaxial sides of the bases of the primary lateral shoots, were suppressed significantly by knock-down of five genes, NtLs, NtB/1, NtREV, VE7, and VE12. Chemical mutation of three of them, $N t L s, N t B / 1$, and NtREV, similarly reduced secondary and "tertiary" lateral shoots but not primary ones. The mutation of $N t L s$ and $N t B / 1$ was backcrossed into an elite variety. The backcross lines were examined for agronomic characteristics in field trials conducted in commercial tobacco production areas. The lines were satisfactory for leaf tobacco production overall and showed good potential as new tobacco varieties.

\section{Conclusion}

Suppression of the five genes reduced only secondary and tertiary lateral shoots in tobacco although similar approaches reduced all branches in other plant species. Nevertheless, the mutant lines might greatly relieve farmers because secondary and tertiary lateral shoots are especially cumbersome because they emerge when farmers are burdened by the labor-intensive leaf harvest.

\section{Background}

Development of lateral shoots, a fundamental process in plants, is regulated in a unique way in every species. In crop species, the harvest quality and productivity are affected directly by how lateral shoot formation is controlled. Therefore, genes involved in axillary meristem development have been studied extensively in various plant species. Mutation in Lateral suppressorgenes $L s$ in tomato [1] and LAS in Arabidopsis [2] inhibit axillary shoot formation during the vegetative phase. Mutation in an orthologue, Monocolum (MOC1), in rice suppressed tillering and decreased rachis branches and spikelets [3]. Mutation in Blind gene strongly suppressed axillary meristem formation in tomato [4,5]. Three Regulator of Axillary Meristems $(R A X)$ genes, RAX1, RAX2, and RAX3, were identified in Arabidopsis as homologous of Blind. The triple recessive mutants almost completely inhibited axillary shoot formation [6, 7]. Blind and $R A X$ genes code for R2R3 Myb transcription factors, which play a central role in the initiation of axillary meristems during the vegetative phase. Blind also controls the axillary meristem initiation in the reproductive phase $[5,6]$. Decapitation of primary shoots did not stimulate outgrowth of axillary shoots in a las mutant or in a rax 1 mutant in Arabidopsis [2, 7]. $L A X 1$ gene in rice and $B A 1$ gene in maize carried bHLH domains and were involved in branching of inflorescence and vegetative shoots $[8,9]$. ROX gene is an orthologue of $L A X 1$ and $B A 1$ in Arabidopsis. It is reportedly involved in lateral shoot formation in the early vegetative stage [10].

Mutation in some genes regulating axillary meristem formation affects the development of main shoot apices. Mutation in the Revolute (REV) gene in Arabidopsis remarkably reduced outgrowth of both rosette and cauline leaves and reduced stimulation of axillary shoots by decapitation of primary shoots $[11,12]$. In addition, rev mutation sometimes arrested development of the primary shoot apical meristems at an early stage. Cup-Shaped Cotyledon (CUC) genes CUC1, CUC2, and CUC3, [13-15] and Lateral Organ Fusion (LOF) gene [16] regulate both organ separation and axillary meristem formation in Arabidopsis. Hairy Meristem (HAM) genes in Petunia [17] and pepper [18] and their homologue, Lost Meristem (LOM) gene, in Arabidopsis [19] play important roles in shoot apical meristem and axillary meristem development. Consequently, ham mutants in Petunia and pepper and lom 1-lom2-lom3 triple mutant in Arabidopsis exhibited premature cession of shoot apex and arrest of axillary shoot development. Mutation in Far-Red Elongated Hypocotyl (FHY3) gene in Arabidopsis inhibited axillary bud outgrowth. Also, rev-fhy 3 double mutation more drastically repressed axillary bud formation than the single recessive mutations [20].

Because tobacco is cultivated for harvesting of the leaves, when plants start to flower, apical portions of stems, mostly inflorescence, are topped off so that leaves well develop and mature for processing. Although tobacco generally exhibits strong apical dominance, this topping releases lateral buds from dormancy. Lateral shoots, which are often called "suckers" in tobacco, start vigorous development. Tobacco farmers must remove these "primary" lateral shoots soon, but then "secondary" lateral shoots grow immediately from the abaxial sides of the bases of the primary lateral shoots. In this manner, lateral shoots emerge sequentially during the cultivation period up to "tertiary" ones. The lateral shoots must be removed manually or suppressed by chemicals, "suckercides", to maintain the leaf harvest quality and quantity. Control of lateral shoots is a labor-intensive and cost-intensive process. Secondary and tertiary lateral shoots are especially cumbersome because, when they emerge, farmers are burdened by another labor-intensive task: leaf harvest. Therefore, tobacco varieties with fewer lateral shoots, especially with fewer secondary and tertiary ones, are highly desired. Nevertheless, breeding including conventional and biotechnological approaches has not remarkably reduced lateral shoots to date.

Little is known about tobacco genes involved in the regulation of axillary shoot development and their functions. However, it is possible that homologues of the genes in other plants mentioned above are present and function similarly in tobacco. For example, a tobacco homologue (GenBank EU935981) of $L s$ was cloned [21], although its function was not studied. In this study, RNA interference (RNAi) of the homologues and a number of other genes preferentially expressed in the axillary meristems was attempted in tobacco. Then, chemically induced mutations were identified for the genes, which showed reduction of lateral shoots in the RNAi screening. Mutant lines were characterized in greenhouse and field trials. 


\section{Results \\ BLAST search}

Two groups of tobacco genes were examined for effects of RNAi knock-down on lateral shoot formation. The first group included homologues of the genes reported as involved in lateral shoot formation in other plants. BLAST search of GenBank and the in-house tobacco cDNA database was conducted for the tobacco homologues (Table 1). Because tobacco is an amphidiploid plant that inherited its genome from N. sylvestris (S-genome) and N. tomentosiformis (Tgenome) [22-24], both S- and T-alleles were identified for each homologue. NtB/ and NtCUC genes were numbered, respectively in descending order of homology with Blind and a tomato homologue (GeneBank HM210879) of the CUC genes.

\section{Rnaseq Study}

Genes that were not studied in other plants might also be involved in the regulation or development of axillary meristems and shoots. Consequently, as the second group, genes that are highly expressed in primordial stages of axillary buds were sought by RNA sequencing. As presented in Fig. 1, tissues were sampled from zones of axillary meristems in the early stage (EA) and the very early stage (VE) and control zones of tobacco plants by laser micro-dissection. RNA was prepared from the tissues and was analyzed using a next generation sequencer: 454 GS FLX. Approximately 900,000 reads, about 400 bp in length on average, were obtained from each tissue sample and were assembled into 38,569 unique genes. The second group of the genes was chosen according to the criteria, (a) read count of a gene in EA or VE was at least 10 times higher than that in the control, (b) the assembled gene was longer than 200 bp, and (c) the gene was of a transcription factor or unknown protein in the annotation. We selected 11 genes highly expressed in EA tissue, EA1-EA11, and 13 genes highly expressed in VE tissue, VE1-VE13 (Table 2).

The counts of the RNA reads assigned to the first group of the genes, the tobacco homologues, are also shown in Table 2. Among them, genes highly and specifically expressed in EA or VE tissue were NtLs and NtB/1. In all three samples, NtREV was highly expressed. The other homologues were either not specifically expressed in EA and VE tissues or were not detectable at all. In the literature, LAS and RAX were highly expressed in the axil of leaf primordia [2, 6, 7]. Also, REV was expressed not only in the early stage of lateral shoot meristem but also in the center of the SAM in inverted-cup-shaped population of the cell [12]. Consequently, at least some of the homologues, $N t L s, N t B / 1$, and $N t R E V$, were similar to their counterparts in other species in the expression patterns.

It is noteworthy that the sequence analysis revealed that $V E 7$, which carried a bHLH domain, was a homologue of ROX gene, which was published after the BLAST s BLAST search described above [10]. In addition, VE12, which carried a NAC domain and which lacked a recognition site of miR164, turned out to be a homologue of CUC3 gene. ROX[10] and its maize orthologue BA1 [9] were expressed at the adaxial boundary of leaf primordia. Expression of $C U C 3$ was detected at the boundaries between leaf primordia and the shoot meristem [25]. Consequently, VE7 and VE12 were similar to the homologues also in the expression patterns. After all, the RNAseq study well supplemented the homologue search.

\section{RNAi knock-down}

A total of 36 trigger sequences, 430 bp in length on average, were designed for RNA interference of the 12 homologues and the 24 genes from RNAseq screening (Supplemental Table S1). For each target, the S-allele and T-allele were compared. A region of high homology was chosen as the trigger so that both alleles would be knocked down effectively by a single trigger sequence. The average homology between the trigger regions of the S- and T-alleles was $95.4 \%$ identity, which was higher than the level of homology sufficient for simultaneous knock-down of two alleles recommended by Parrish et al. [26]. In this process, it was possible to find less conserved regions, less than $70 \%$ identity, among NtB/genes and among NtCUC genes. Therefore, it was likely that a trigger was able to knock down the S-T pair of the target gene specifically.

The RNAi genes were introduced into tobacco 'Petit Havana SR-1'. The T1 progeny of three single locus transformants for each construct were grown in a greenhouse and were examined for the target gene expression. The target genes were well suppressed (Table 2) except for VE3 transformants, in which the target gene was expressed higher than the null segregants, for reasons that remain unknown. Results showed that all transgenic plants produced as many and as much primary lateral shoots as the null segregants (Table 2). Therefore, the RNAi of no target suppressed primary lateral shoots. However, the secondary lateral shoots were well suppressed in number and/or weight in the transformants of five constructs: NtLs, NtB/1, NtREV, VE7, and VE12. For example, although a secondary lateral bud was visible at the base of the abaxial side of a primary lateral shoot of a wild type plant (Fig. 2a), there was none at the position of an RNAi knock-down plant for NTLs (Fig. 2b). After removal of the secondary lateral shoots, no more lateral shoot formation was observed during greenhouse testing. Therefore, these five genes were studied further. However, the transformants of EA5 and VE6 produced more secondary lateral shoots than the null segregants.

\section{Chemically induced mutants}

The EMS mutant library of tobacco 'Tsukuba 1' was screened for nonsense mutations of NtLS, NtB/1, NtREV, VE7 and VE12. As depicted in Fig. 3, the following mutations were identified and mapped: two each in NtLs-S and NtB/1-S and one each in NtLs-T, NtB/1-T, NtRev-S, and NtRev-T. Also, mutations in Sand T- genomes were combined by crossing the mutants, producing double recessive lines designated as SAS lines (Table 3). Because nonsense mutations were not identified in VE7 and VE12, these genes were not studied further.

The SAS lines, 15 plants each, were planted in an experimental field and were examined for lateral shoot development. Again, primary lateral shoots were not suppressed significantly in the mutant lines (Table 4). Then, the number and weight of secondary lateral shoots were well suppressed. Those of tertiary ones were suppressed nearly completely. Therefore, the observation made in the RNAi lines in a greenhouse was faithfully reproduced in the EMS mutants in the field trial. It is particularly interesting that the position of the primary lateral shoots was shifted upward in the SAS-Is and SAS-b/1 mutant lines (Fig. 2d and 2f) 
in the field trial and also in a greenhouse (data not shown). No such shift was observed in the RNAi lines in the greenhouse (Fig. 2b). The positions of the secondary and tertiary lateral shoots in the mutant lines and the RNAi lines were unchanged. Only the position of the primary lateral shoots was affected, for reasons that remain unclear.

\section{Field trials in commercial production areas}

Field trials in commercial production areas were conducted to examine agronomic characteristics of the mutant lines. The /s-1 and $b / 1-1$ mutations were introduced into a flue-cured tobacco variety, 'Coker319', widely grown in Japan by backcross breeding (Table 3). Because of space limitations, the rev mutation was not tested.

Results of the trials of the backcross lines are presented in Table 5 for Coker319-/s-1 at four locations and Coker319-b/1- 1 at two locations. A plot of 10 plants was replicated twice for each line at all locations. Overall, the mutant lines did not differ much from the original 'Coker319' in leaf yield or the other measurements, although small but statistically significant differences were found as explained hereinafter. Coker319-/s-1 was earlier in days to flower, higher in plant height, larger in leaf length, larger in leaf width, with darker leaf color than 'Coker319' at one or more locations. Coker319-b/1-1 had lower plant height, more numerous leaves, smaller leaf width at 1/2 from the top, and lighter leaf color than 'Coker319' at one or two locations. Therefore, no problems were found in the mutant lines as raw materials. The continuation of the trials was decided. In addition, reduction of lateral shoot developments, especially of secondary and tertiary lateral shoots, was observed. The labor necessary for removal of lateral shoots of Coker319-/s-1 was lower by $52 \%$ than that of 'Coker319' in a preliminary survey (Supplemental Table S2).

\section{Other characteristics of the mutants}

During the course of the study, some other phenotypic alterations were noticed. Leaves of 4-5-week-old Coker319-/s-1 plants occasionally turned yellow in nursery boxes (Fig. 2h). It was then confirmed that the same phenotype was observed in a growth chamber when 4-5-week-old Coker319-/s-1 plants were grown at $15^{\circ} \mathrm{C}$, although they were normal at $28^{\circ} \mathrm{C}$. Results show that the Coker319-/s- 1 leaf color was cold-sensitive. Some plants were probably exposed to low temperatures in the nursery boxes. However, yellowed plants recovered quickly and showed no growth delay.

Petals of most of the flowers of SAS-Is-1, SAS-Is-2, and Coker319-/s-1 plants were split (Fig. 2i) in greenhouses and in the field. In severe cases, pistils and stamens were completely exposed (Fig. 2j). Seed yield from these flowers decreased considerably, by $35 \%$ and $55 \%$ of the wild type lines, respectively, by natural and hand pollination (Supplemental Fig. S1).

Flowers of Coker319-b/1-1 plants were 73\% fewer than those of the wild type lines in the field trials (Supplemental Fig. S2). The seed yield from the flowers of Coker319-b/1-1 was $72 \%$ of that of 'Coker319' (Supplemental Fig. S1).

\section{Discussion}

Genetic reduction of lateral shoots was attempted in tobacco. Two groups of tobacco genes were examined for effects of RNAi knock-down on lateral shoot formation. One source of the candidate genes was homologues of the genes reported as involved in the formation of lateral shoots in other plants. Such genes have been studied most extensively in Arabidopsis. The following eight genes or gene families were identified: $L A S[2], R A X$ family [6, 7], $R O X$ [10], $R E V$ [11, 12], CUC family [13-15], LOF [16], LOM [19], and FHY3 [20]. In tobacco, the BLAST search found homologues of seven of them, excluding ROX, which was reported after the BLAST search was performed. Then VE7, a homologue of ROX, was found from another source, genes highly expressed in primordial stages of axillary buds in tobacco in the RNA seq analysis. A homologue of CUC3, VE12, was also found from the second source. Therefore, tobacco and Arabidopsis apparently shared similar sets of the genes.

Knock-down of five genes of the set in tobacco, NtLs, NtB/1, NtREV, VE7, and VE12, and chemically induced mutation of three of them, NtLs, NtBI1, and NtREV, caused suppression of the secondary lateral shoots. The NtLs, NtBI1, NtREV, VE7, and VE12 were respective homologues of LAS, RAX1, REV, ROX, and CUC3. Their reported expression patterns were consistent with results obtained for the RNAseq study. In addition, mutations in $L A S$ [2], $L s$ [1] in tomato orthologue, and $N t L s$ all affected flower morphology. Mutations both in Blind [5], which was a tomato orthologue of RAX1, and NtB/1 reduced flower numbers. Therefore, NtLS, NtBI1, NtREV, VE7, and VE12 might be considered respectively as orthologues of LAS, RAX1, REV, ROX, and CUC3.

However, some characteristics of the knock-down and mutant tobacco were not reported in other plants. Primary lateral shoots were not suppressed, whereas secondary and tertiary ones were well suppressed in tobacco. In addition, the positions of primary lateral shoots in the tobacco mutants of NtLs and NtB/1 shifted upward. The mechanisms for differences between the plant species remain unclear, but the presence of the difference is not surprising because diverse ways of regulating lateral branching must be a key factor for a wide variety of plant shapes.

The other tobacco homologues were not effective in the knock-down study. The expression of these genes was either not detected in or was not specific to the primordial stages of axillary buds. They were probably not functional counterparts of the homologues of the other plants.

The second source of the candidate genes examined were genes that were highly expressed in the primordial stages of axillary buds. Quite a few, 24, genes were chosen from the RNAseq analysis. They were highly expressed in and specific to EA or VE tissues. However, except for VE7 and VE12 described above, knock-down of no gene caused significant reduction of lateral shoots. These genes might not be playing crucial regulatory roles in lateral shoot development. Alternatively, their functions might be redundant in tobacco.

Two mutant tobacco lines under the genetic background of variety 'Coker319' were examined in field trials at commercial tobacco production sites. A general conclusion is that these lines were satisfactory for leaf tobacco production and that trials are worth repeating. Regarding the reduction of secondary and 
tertiary lateral shoots, the labor required for removal of lateral shoots in a preliminary survey was observed. Although cold sensitivity at the seedling stage and some abnormalities of flowers that decreased seed yield were observed in the mutants of $N t L s$, these characteristics were not regarded as difficulties likely to affect leaf tobacco production practices.

Therefore, the 'Coker319' derivatives showed good potential as new tobacco varieties. They can relieve farmers from the difficult labor necessary for control of secondary and tertiary lateral shoots. In addition, breeding of other lines with mutations in NtLs or NtBI1, the combination of these mutations, and further studies of $N t L s, N t B / 1, N t R E V, V E 7$, and VE12 genes are among important future tasks.

\section{Conclusions}

Development of tobacco lines with reduced lateral shoots, which is strongly desired by tobacco farmers, was attempted. Indeed, RNA interference of five genes and the chemical mutation of three of them significantly suppressed lateral shoot development in tobacco in a greenhouse and in field tests conducted at an experimental site. Two mutant lines were evaluated for agronomic performance in field trials conducted at commercial production sites. Although primary lateral shoots did not decrease in the lines, the reduction of secondary and tertiary lateral shoots, which are especially cumbersome in tobacco production, was an important step forward.

\section{Methods}

\section{BLAST analysis}

Query genes for BLAST search of NCBI BLAST database (http://blast.ncbi.nlm.nih.gov/Blast.cgi) and a database of tobacco cDNA sequences accumulated by a number of in-house studies are shown in Table 1.

\section{RNA sequencing (RNAseq)}

Apical tissues of shoots were collected from seedlings of tobacco 'Petit Havana SR-1' at 29-37 days after germination and were fixed in an ice-cold 3:1 solution of ethanol and acetic acid. Protocols for preparation of paraffin-embedded sections and laser-microdissection were described by Takahashi et al. [27]. Total RNA was extracted using a PicoPure ${ }^{\mathrm{TM}}$ RNA isolation Kit (Thermo Fisher Scientific Inc.) and was examined using an Agilent 2100 Bioanalyzer and an RNA 6000 Pico kit (Agilent Technologies Inc.). Also, cDNA was synthesized using an oligo-dT primer with the T7 promoter sequence; then antisense RNA was transcribed by T7 RNA polymerase. Again, CDNA was synthesized from the antisense RNA using random primers. The secondary cDNA was sequenced by 454 GS FLX. The obtained reads were assembled by Newbler ver. 2.6.

\section{Cloning of genes}

After coding sequence information was obtained from databases (GenBank and the in-house cDNA database), 5' or 3'-RACE was performed with a SMARTer RACE cDNA Amplification Kit (Clontech) to obtain full-length cDNA clones of the coding sequences. RNAs were extracted from a dormant axillary bud, shoot apex, flower bud, or root (Magtraton ${ }^{\circledR}$; Precision System Science Co., Ltd.). Amplified fragments were sequenced with a capillary sequencer (3730xI DNA Analyzer, Thermo Fisher Scientific Inc.) using a BigDye ${ }^{\circledR}$ V3.1 Cycle Sequencing Kit (Thermo Fisher Scientific Inc.). DNA manipulations in this study were performed according to standard procedures [28].

\section{RNAi}

Trigger sequences were amplified using the primers listed in Supplemental Table S1 from the cloned cDNAs or RNA isolated from dormant axillary buds, shoot apices, flower buds or roots of tobacco 'Petit Havana SR-1' with Magtraton ${ }^{\circledR}$ technology. The amplified fragments were cloned into pENTR ${ }^{\mathrm{TM}} / \mathrm{D}$-TOPO vector (Takara Bio) and were then introduced into a modified pHellsgate12 binary vector [29], which had an expression cassette of a green fluorescence gene driven by the CaMV 35S promoter. The binary vectors were introduced into Agrobacterium tumefaciens strain LBA4404. Tobacco 'Petit Havana SR-1' was transformed with the resultant strains. Transgene locus number was determined by segregation ratios of the green fluorescence in the T1 generation. Expression level of the target genes was measured using quantitative PCR. RNAs were prepared with Magtration ${ }^{\circledR}$ technology from leaves, roots or aboveground tissues excluding leaves. The cDNAs were synthesized using PrimeScript RT reagent kit with gDNA Eraser (Takara Bio). Real-time PCR was conducted from the cDNAs using StepOnePlus (Thermo Fisher Scientific Inc.) and TaqMan Fast Advanced Master Mix (Thermo Fisher Scientific Inc.). Primers and probes are presented in Supplemental Table S3. Elongation factor-1a (AF120093) was used as a reference gene.

\section{Chemically induced mutants}

A library of tobacco 'Tsukuba 1' mutated with ethyl methanesulfonate (EMS) constructed by Tajima et al. [30] was screened using the protocol described by Takakura et al. [31] with primers listed in Supplemental Table S4.

\section{Plant cultivation}

Tobacco plants were grown in soil pots $(12 \mathrm{~cm})$ in a greenhouse under natural day length at $25^{\circ} \mathrm{C}$ or in a growth chamber under the cycle of $12 \mathrm{~h}$ light at $25^{\circ} \mathrm{C}$ and $12 \mathrm{~h}$ dark at $18^{\circ} \mathrm{C}$, in an experimental field at the Leaf Tobacco Research Center of Japan Tobacco Inc., or in fields in commercial tobacco production areas in southwest Japan, according to the respective standard cultivation practice. When flower buds emerged, the plants were topped off. The lateral shoots were removed and measured weekly for five weeks in the growth chamber or for eight weeks in the field after the topping. Leaf color was scored using a Leaf Color Chart 2019A for tobacco (Fujihira Industry, Supplemental Table S5). 


\section{Abbreviations}

LS, LAS

LATERAL SUPPRESSOR

MOC1

MONOCOLUM 1

RAX

REGULATOR of AXILLARY MERISTEMS

MYB

Myeloblastosis

$\angle A X$

LAX PANICLE

$B A 1$

BARREN STALK 1

bHLH

basic helix-loop-helix

ROX

REGULATOR OF AXILLARY MERISTEM FORMATION

REV

REVOLUTE

CUC

CUP-SHAPED COTYLEDON

LOF

LATERAL ORGAN FUSION

HAM

HAIRY MERISTEM

LOM

LOST MERISTEM

FHY3

FAR-RED ELONGATED HYPOCOTYL3

RNAi

RNA interference

NCBI

National Center for Biotechnology Information

BLAST

Basic Local Alignment Search Tool

$B I$

$B L I N D$

RNA-seq

Ribonucleic acid sequencing

SAM

Shoot apical meristem

NAC

for petunia NAM and Arabidopsis ATAF1, ATAF2, and CUC2

EMS

Ethyl methanesulfonate

RACE

Rapid Amplification of cDNA Ends

CaMV

Cauliflower mosaic virus

SD

standard deviation

\section{Declarations}

\section{Acknowledgments}

The authors acknowledge Dr Toshihiko Komari his kind, critical, and careful reading of the manuscript. They acknowledge Nanae Sato, Sayaka Koike, and Akiko Takahashi for their excellent technical assistance. 
$\mathrm{KH}, \mathrm{SeS}, \mathrm{TaN}$, and ShS, designed the research and experiments. KH, SeS, MA, YN, TaN, TK, TsN, and ShS performed the experiments. KH, SeS, and ShS regularly discussed the research progress and developed the research strategy. $\mathrm{KH}$ and ShS wrote the manuscript.

\section{Funding}

Japan Tobacco Inc., with which all authors are affiliated, is the sole funder of the research.

\section{Availability of data and materials}

The datasets generated or analyzed during the current study are available from the corresponding author on reasonable request.

\section{Declarations}

\section{Ethics approval and consent to participate}

Not applicable.

\section{Conflict of interest}

All authors are affiliated with Japan Tobacco Inc., a funder of this study.

\section{Competing interests}

The authors declare that they have no competing interests.

\section{References}

1. Schumacher K, Schmitt T, Rossberg M, Schmitz G, Theres K. The Lateral suppressor (Ls) gene of tomato encodes a new member of the VHIID protein family. Proc Natl Acad Sci U S A. 1999;96:290-5.

2. Greb T, Clarenz O, Schafer E, Muller D, Herrero R, Schmitz G, Theres K. Molecular analysis of the LATERAL SUPPRESSOR gene in Arabidopsis reveals a conserved control mechanism for axillary meristem formation. Genes Dev. 2003;17:1175-87.

3. Li X, Qian Q, Fu Z, Wang Y, Xiong G, Zeng D, Wang X, Liu X, Teng S, Hiroshi F, et al. Control of tillering in rice. Nature. 2003;422:618-21.

4. Mapelli S, Kinet JM. Plant growth regulator and graft control of axillary bud formation and development in the TO-2 mutant tomato. Plant Growth Regul. 1992;11:385-90.

5. Schmitz G, Tillmann E, Carriero F, Fiore C, Cellini F, Theres K. The tomato Blind gene encodes a MYB transcription factor that controls the formation of lateral meristems. Proc Natl Acad Sci U S A. 2002;99:1064-9.

6. Keller T, Abbott J, Moritz T, Doerner P. Arabidopsis REGULATOR OF AXILLARY MERISTEMS1 controls a leaf axil stem cell niche and modulates vegetative development. Plant Cell. 2006;18:598-611.

7. Müller D, Schmitz G, Theres K. Blind homologous R2R3 Myb genes control the pattern of lateral meristem initiation in Arabidopsis. Plant Cell. 2006;18:586-97.

8. Komatsu K, Maekawa M, Ujiie S, Satake Y, Furutani I, Okamoto H, Shimamoto K, Kyozuka J. LAX and SPA: major regulators of shoot branching in rice. Proc Natl Acad Sci U S A. 2003;100:11765-70.

9. Gallavotti A, Zhao Q, Kyozuka J, Meeley RB, Ritter MK, Doebley JF, Pè ME, Schmidt RJ. The role of barren stalk1 in the architecture of maize. Nature. 2004;432:630-5.

10. Yang F, Wang Q, Schmitz G, Müller D, Theres K. The bHLH protein ROX acts in concert with RAX1 and LAS to modulate axillary meristem formation in Arabidopsis. Plant J. 2012;71:61-70.

11. Talbert PB, Adler HT, Parks DW, Comai L. The REVOLUTA gene is necessary for apical meristem development and for limiting cell divisions in the leaves and stems of Arabidopsis thaliana. Development. 1995;121:2723-35.

12. Otsuga D, DeGuzman B, Prigge MJ, Drews GN, Clark SE. REVOLUTA regulates meristem initiation at lateral positions. Plant J. 2001;25:223-36.

13. Aida M, Ishida T, Fukaki H, Fujisawa H, Tasaka M. Genes involved in organ separation in Arabidopsis: an analysis of the cup-shaped cotyledon mutant. Plant Cell. 1997;9:841-57.

14. Aida M, Ishida T, Tasaka M. Shoot apical meristem and cotyledon formation during Arabidopsis embryogenesis: interaction among the CUP-SHAPED COTYLEDON and SHOOT MERISTEMLESS genes. Development. 1999;126:1563-70.

15. Takada S, Hibara K, Ishida T, Tasaka M. The CUP-SHAPED COTYLEDON1 gene of Arabidopsis regulates shoot apical meristem formation. Development. 2001;128:1127-35.

16. Lee DK, Geisler M, Springer PS. LATERAL ORGAN FUSION1 and LATERAL ORGAN FUSION2 function in lateral organ separation and axillary meristem formation in Arabidopsis. Development. 2009;136:2423-32.

17. Stuurman J, Jäggi F, Kuhlemeier C. Shoot meristem maintenance is controlled by a GRAS-gene mediated signal from differentiating cells. Genes Dev. 2002;16:2213-8.

18. David-Schwartz R, Borovsky Y, Zemach H, Paran I. CaHAM is autoregulated and regulates CaSTM expression and is required for shoot apical meristem organization in pepper. Plant Sci. 2013;203-204:8-16. 
19. Schulze S, Schäfer BN, Parizotto EA, Voinnet O, Theres K. LOST MERISTEMS genes regulate cell differentiation of central zone descendants in Arabidopsis shoot meristems. Plant J. 2010;64:668-78.

20. Stirnberg P, Zhao S, Williamson L, Ward S, Leyser O. FHY3 promotes shoot branching and stress tolerance in Arabidopsis in an AXR1-dependent manner. Plant J. 2012;71:907-20.

21. Wang W, Tai S, Wang L, Liu G-s, Liu F-x. Gao X-m, Sun Y-h: Construction of RNAi Vector of NtLS Gene and its Transformation in Tobacco. Chinese Tobacco Science. 2011;32:31-5.

22. Riechers DE, Timko MP. Structure and expression of the gene family encoding putrescine N-methyltransferase in Nicotiana tabacum: new clues to the evolutionary origin of cultivated tobacco. Plant Mol Biol. 1999;41:387-401.

23. Murad L, Lim KY, Christopodulou V, Matyasek R, Lichtenstein CP, Kovarik A, Leitch AR. The origin of tobacco's T genome is traced to a particular lineage within Nicotiana tomentosiformis (Solanaceae). Am J Bot. 2002;89:921-8.

24. Gavilano LB, Coleman NP, Bowen SW, Siminszky B. Functional analysis of nicotine demethylase genes reveals insights into the evolution of modern tobacco. J Biol Chem. 2007;282:249-56.

25. Hibara K, Karim MR, Takada S, Taoka K, Furutani M, Aida M, Tasaka M. Arabidopsis CUP-SHAPED COTYLEDON3 regulates postembryonic shoot meristem and organ boundary formation. Plant Cell. 2006;18:2946-57.

26. Parrish S, Fleenor J, Xu S, Mello C, Fire A. Functional anatomy of a dsRNA trigger: differential requirement for the two trigger strands in RNA interference. Mol Cell. 2000;6:1077-87.

27. Takahashi H, Kamakura H, Sato Y, Shiono K, Abiko T, Tsutsumi N, Nagamura Y, Nishizawa NK, Nakazono M. A method for obtaining high quality RNA from paraffin sections of plant tissues by laser microdissection. J Plant Res. 2010;123:807-13.

28. Sambrook JF, Russell DW. Molecular Cloning: A Laboratory Manual (3-Volume Set). Cold Spring Harbor: Cold Spring Harbor Laboratory Press; 2001.

29. Wesley SV, et al. Construct design for efficient, effective and high-throughput gene silencing in plants. Plant J. 2001;27:581-90.

30. Tajima T, Sato S, Hiyoshi T. Construction of mutant panel in Nicotiana tabacum L. Ann Phytopathol Soc Jpn. $2011 ; 77$.

31. Takakura Y, Udagawa H, Shinjo A, Koga K. Mutation of a Nicotiana tabacum L. eukaryotic translation-initiation factor gene reduces susceptibility to a resistance-breaking strain of Potato virus Y. Mol Plant Pathol. 2018;19:2124-33.

\section{Tables}

Table 1. Tobacco homologues of the genes in other species involved in regulation or development of axillary meristems and shoots

\begin{tabular}{|c|c|c|c|}
\hline \multirow[t]{2}{*}{ Query gene (Species, GenBank accession) for BLAST search } & \multirow[t]{2}{*}{$\begin{array}{l}\text { Tobacco } \\
\text { homologue }\end{array}$} & \multicolumn{2}{|c|}{$\begin{array}{l}\text { Accession number in GenBank or Sol Genomics } \\
\text { Network }^{1}\end{array}$} \\
\hline & & S-allele & T-allele \\
\hline Ls (Tomato, AF098674) & NtLs & EU935581 & AM848584 \\
\hline \multirow[t]{3}{*}{ MYB domain of Blind (Tomato, AF426174.1) } & $N t B / 1$ & Nitab4.5_0001442g0010.1 & Nitab4.5_0004993g0020.1 \\
\hline & $N t B / 2$ & Nitab4.5_0001050g0010.1 & Nitab4.5_0001163g0150.1 \\
\hline & $N t B / 3$ & Nitab4.5_0007679g0010.1 & Nitab4.5_0000578g0120.1 \\
\hline REV (Tomato, BT013577 and Arabidopsis, AF233592) & NtREV & JQ686937 & Nitab4.5_0004624g0050.1 \\
\hline \multirow{4}{*}{$\begin{array}{l}\text { NAC domains of CUC genes (Arabidopsis, NM_112380, NM_124774, and } \\
\text { NM_106292) }\end{array}$} & NtCUC1 & Nitab4.5_0007278g0010.1 & Nitab4.5_0000568g0080.1 \\
\hline & NtCUC2 & Nitab4.5_0008840g0010.1 & Nitab4.5_0005914g0020.1 \\
\hline & NtCUC3 & Nitab4.5_0004286g0020.1 & Nitab4.5_0007189g0080.1 \\
\hline & NtCUC4 & XM_016587094 & XM_016602286.1 \\
\hline FHY3 (Arabidopsis, NM_001125201) & NtFHY3 & Nitab4.5_0002232g0050.1 & XM_016593996.1 \\
\hline LOM (Arabidopsis, NM_130079.3) & NtLOM1 & Nitab4.5_0011278g0010.1 & XM_016625781.1 \\
\hline LOF (Arabidopsis, NM_001160897.2) & NtLOF1 & XM_016601029.1 & XM_016602279.1 \\
\hline
\end{tabular}

${ }^{1}$ Homologues originally found from the in-house database were later published in Sol Genomics Network database by other research groups. Therefore, the accession numbers (Nitab numbers) in the public database are shown.

Table 2. Expression of the genes in the early axillary meristem tissues and effects of RNA interference 


\begin{tabular}{|c|c|c|c|c|c|c|c|c|}
\hline \multirow[t]{3}{*}{ Gene } & \multirow{2}{*}{\multicolumn{3}{|c|}{$\begin{array}{l}\text { RNAseq count } \\
\text { (Reads Per Kilobase Million) }\end{array}$}} & \multicolumn{5}{|c|}{ Effect of RNAi measured relative to null segregants \pm SD } \\
\hline & & & & \multirow[t]{2}{*}{ RNA expression } & \multicolumn{2}{|c|}{ Primary lateral shoots } & \multicolumn{2}{|c|}{ Secondary lateral shoots } \\
\hline & EA & VE & Control & & Number & Weight & Number & Weight \\
\hline$N t L s$ & 1.16 & 62.97 & 1.14 & $0.50 \pm 0.08$ & $1.00 \pm 0.00$ & $1.28 \pm 0.15$ & $0.13 \pm 0.09 * *$ & $0.1 \pm 0.06^{\star \star}$ \\
\hline$N+B / 1$ & 12.80 & 108.85 & 8.43 & $0.31 \pm 0.05$ & $1.02 \pm 0.06$ & $1.21 \pm 0.18$ & $0.00 \pm 0.00 * \star$ & $0.00 \pm 0.00 * *$ \\
\hline$N t B / 2$ & ND & ND & ND & $0.19 \pm 0.04$ & $1.00 \pm 0.00$ & $1.13 \pm 0.15$ & $1.00 \pm 0.10$ & $1.46 \pm 1.65$ \\
\hline$N+B / 3$ & 11.88 & 23.20 & 10.26 & $0.30 \pm 0.09$ & $1.00 \pm 0.00$ & $1.45 \pm 0.48$ & $0.88 \pm 0.46$ & $0.77 \pm 0.12$ \\
\hline NtREV & 110.74 & 167.99 & 305.61 & $0.62 \pm 0.10$ & $1.00 \pm 0.00$ & $0.98 \pm 0.09$ & $0.06 \pm 0.10 * *$ & $0.03 \pm 0.04^{\star \star}$ \\
\hline NtCUC1 & 4.24 & 10.32 & 8.38 & $0.16 \pm 0.07$ & $1.00 \pm 0.00$ & $1.05 \pm 0.12$ & $0.91 \pm 0.18$ & $0.57 \pm 0.10$ \\
\hline NtCUC2 & ND & ND & ND & $0.53 \pm 0.04$ & $1.00 \pm 0.00$ & $1,14 \pm 0.26$ & $0.97 \pm 0.12$ & $1.06 \pm 0.4$ \\
\hline NtCUC3 & ND & ND & ND & $0.17 \pm 0.10$ & $1.00 \pm 0.00$ & $0.96 \pm 0.17$ & $1.08 \pm 0.01$ & $0.71 \pm 0.26$ \\
\hline NtCUC4 & ND & ND & ND & $0.69 \pm 0.03$ & $1.00 \pm 0.00$ & $0.91 \pm 0.04$ & $1.05 \pm 0.09$ & $0.75 \pm 0.19$ \\
\hline$N t F H Y 3$ & 23.49 & 36.90 & 46.59 & $0.31 \pm 0.17$ & $1.00 \pm 0.00$ & $0.94 \pm 0.08$ & $1.03 \pm 0.11$ & $1.12 \pm 0.62$ \\
\hline NtLOM1 & 36.69 & 49.15 & 65.87 & $0.38 \pm 0.06$ & $1.00 \pm 0.00$ & $1.09 \pm 0.15$ & $1.72 \pm 1.97$ & $2.56 \pm 3.99$ \\
\hline NtLOF1 & 30.27 & 60.57 & 25.56 & $0.38 \pm 0.11$ & $1.01 \pm 0.02$ & $1.67 \pm 0.15$ & $0.28 \pm 0.30$ & $0.64 \pm 0.58$ \\
\hline$E A 1$ & 32.93 & 2.30 & ND & $0.09 \pm 0.03$ & $1.00 \pm 0.00$ & $1.14 \pm 0.16$ & $0.97 \pm 0.15$ & $1.21 \pm 0.34$ \\
\hline$E A 2$ & 20.13 & 9.70 & 1.48 & $0.28 \pm 0.16$ & $1.00 \pm 0.00$ & $1.17 \pm 0.35$ & $1.34 \pm 0.82$ & $1.65 \pm 1.59$ \\
\hline$E A 3$ & 22.56 & 1.77 & ND & $0.16 \pm 0.03$ & $1.00 \pm 0.00$ & $0.93 \pm 0.09$ & $0.68 \pm 0.16$ & $1.42 \pm 1.53$ \\
\hline EA4 & 43.22 & 2.50 & 1.19 & $0.35 \pm 0.17$ & $1.00 \pm 0.00$ & $1.16 \pm 0.28$ & $1.14 \pm 0.41$ & $1.09 \pm 0.38$ \\
\hline EA5 & 14.41 & ND & ND & $0.10 \pm 0.04$ & $1.00 \pm 0.00$ & $1.04 \pm 0.07$ & $4.05 \pm 2.21$ & $18.88 \pm 28.34$ \\
\hline$E A 6$ & 22.78 & ND & ND & $0.80 \pm 0.22$ & $1.00 \pm 0.00$ & $1.01 \pm 0.26$ & $1.04 \pm 0.27$ & $1.30 \pm 0.80$ \\
\hline EA7 & 12.14 & ND & ND & $0.10 \pm 0.01$ & $1.00 \pm 0.00$ & $1.02 \pm 0.17$ & $0.83 \pm 0.76$ & $0.77 \pm 0.70$ \\
\hline$E A 8$ & 43.57 & ND & 1.47 & $0.02 \pm 0.02$ & $1.00 \pm 0.00$ & $1.08 \pm 0.36$ & $1.14 \pm 0.11$ & $1.21 \pm 0.2$ \\
\hline$E A 9$ & 14.03 & ND & ND & $0.17 \pm 0.12$ & $1.00 \pm 0.00$ & $0.95 \pm 0.17$ & $1.10 \pm 0.77$ & $1.16 \pm 0.74$ \\
\hline EA10 & 51.93 & 8.72 & 1.25 & $0.43 \pm 0.08$ & $1.00 \pm 0.00$ & $1.33 \pm 0.16$ & $1.59 \pm 0.99$ & $0.96 \pm 0.21$ \\
\hline EA11 & 19.63 & 1.70 & 1.62 & $0.56 \pm 0.08$ & $1.00 \pm 0.00$ & $0.98 \pm 0.27$ & $0.98 \pm 0.09$ & $1.52 \pm 0.92$ \\
\hline VE1 & 39.71 & 49.37 & 3.79 & $0.58 \pm 0.06$ & $1.00 \pm 0.00$ & $0.99 \pm 0.30$ & $1.10 \pm 0.16$ & $1.27 \pm 0.51$ \\
\hline VE2 & 26.70 & 32.21 & 0.69 & $0.51 \pm 0.02$ & $1.00 \pm 0.00$ & $1.04 \pm 0.12$ & $1.09 \pm 0.17$ & $0.95 \pm 0.54$ \\
\hline VE3 & 6.51 & 49.14 & ND & $1.51 \pm 0.05$ & $1.00 \pm 0.00$ & $1.16 \pm 0.21$ & $1.02 \pm 0.45$ & $0.97 \pm 0.35$ \\
\hline VE4 & ND & 10.94 & ND & $0.32 \pm 0.04$ & $1.00 \pm 0.00$ & $1.27 \pm 0.31$ & $0.80 \pm 0.38$ & $0.95 \pm 0.64$ \\
\hline VE5 & 20.88 & 79.27 & 3.12 & $0.41 \pm 0.06$ & $1.00 \pm 0.00$ & $1.00 \pm 0.17$ & $1.3 \pm 0.74$ & $1.41 \pm 0.84$ \\
\hline VE6 & 2.61 & 31.26 & ND & $0.35 \pm 0.14$ & $1.00 \pm 0.00$ & $0.73 \pm 0.12$ & $2.06 \pm 0.56$ & $4.57 \pm 3.20$ \\
\hline VE7 & ND & 73.71 & 4.97 & $0.08 \pm 0.02$ & $1.00 \pm 0.00$ & $1.49 \pm 0.30$ & $0.33 \pm 0.09 * *$ & $0.26 \pm 0.11^{\star *}$ \\
\hline VE8 & 5.93 & 13.38 & ND & $0.14 \pm 0.06$ & $1.00 \pm 0.00$ & $1.01 \pm 0.28$ & $1.41 \pm 0.43$ & $0.87 \pm 0.39$ \\
\hline VE9 & 5.06 & 14.07 & ND & $0.62 \pm 0.08$ & $1.00 \pm 0.00$ & $0.98 \pm 0.10$ & $1.03 \pm 0.16$ & $0.90 \pm 0.12$ \\
\hline VE10 & 9.42 & 21.58 & 1.86 & $0.42 \pm 0.04$ & $1.00 \pm 0.00$ & $0.97 \pm 0.25$ & $1.16 \pm 0.62$ & $1.79 \pm 1.29$ \\
\hline VE11 & ND & 13.62 & ND & $0.70 \pm 0.07$ & $1.00 \pm 0.00$ & $1.07 \pm 0.28$ & $0.58 \pm 0.56$ & $0.42 \pm 0.43$ \\
\hline VE12 & 19.79 & 133.95 & 11.71 & $0.18 \pm 0.06$ & $1.00 \pm 0.00$ & $1.22 \pm 0.12$ & $0.29 \pm 0.41$ & $0.05 \pm 0.08$ ** \\
\hline VE13 & 5.68 & 19.24 & ND & $0.14 \pm 0.05$ & $1.00 \pm 0.00$ & $1.03 \pm 0.21$ & $1.03 \pm 0.12$ & $1.86 \pm 0.94$ \\
\hline
\end{tabular}

**: Significantly different from null segregants at the $1 \%$ level, assuming that the means of the relative values were samples from normal distributions ND: Not detectable 


\begin{tabular}{|c|c|c|c|}
\hline Line code & Target gene & Genotype & Generation \\
\hline SAS-Is-1 & $N t L s$ & s1s1/t1t1 & $(\mathrm{M} 2 \times \mathrm{M} 2) \mathrm{F} 3$ \\
\hline SAS-Ls-1 & NtLs & $S S / T T$ & $(\mathrm{M} 2 \times \mathrm{M} 2) \mathrm{F} 3$, wild type segregant \\
\hline Coker319-/s-1 & $N t L s$ & s1s1/t1t1 & $(($ Coker319 x (M2 x M2) F1) x Coker319) BC6F3 \\
\hline SAS-Is-2 & $N t L s$ & $s 2 s 2 / t 1 t 1$ & $(\mathrm{M} 2 \times \mathrm{M} 2) \mathrm{F} 3$ \\
\hline SAS-Ls-2 & $N t L s$ & $S S / T T$ & $(\mathrm{M} 2 \times \mathrm{M} 2) \mathrm{F} 3$, wild type segregant \\
\hline SAS-b/1-1 & $N t B / 1$ & $s 2 s 2 / t 1 t 1$ & $(\mathrm{M} 2 \times \mathrm{M} 2) \mathrm{F} 3$ \\
\hline SAS-B/1-1 & $N t B / 1$ & $S S / T T$ & $(\mathrm{M} 2 \times \mathrm{M} 2) \mathrm{F} 3$, wild type segregant \\
\hline Coker319-b/1-1 & $N t B / 1$ & $s 2 s 2 / t 1 t 1$ & $(($ Coker319 $\times(\mathrm{M} 2 \times \mathrm{M} 2) \mathrm{F} 1) \times$ Coker319) BC6F3 \\
\hline SAS-b/1-2 & $N t B / 1$ & s1s1t1t1 & $(\mathrm{M} 2 \times \mathrm{M} 2) \mathrm{F} 3$ \\
\hline SAS-B/1-2 & $N t B / 1$ & $S S / T T$ & $(\mathrm{M} 2 \times \mathrm{M} 2) \mathrm{F} 3$, wild type segregant \\
\hline SAS-rev & NtREV & $s 1 s 1 / t 1 t 1$ & $(\mathrm{M} 2 \times \mathrm{M} 2) \mathrm{F} 3$ \\
\hline SAS-REV & NtREV & $S S / T T$ & $(\mathrm{M} 2 \times \mathrm{M} 2) \mathrm{F} 3$, wild type segregant \\
\hline
\end{tabular}

Table 4. Lateral shoot production in field trials at an experimental site

\begin{tabular}{|c|c|c|c|c|c|c|c|}
\hline \multirow[t]{2}{*}{ Trial No. } & \multirow[t]{2}{*}{ Line code } & \multicolumn{2}{|c|}{ Primary lateral shoots } & \multicolumn{2}{|c|}{ Secondary lateral shoots } & \multicolumn{2}{|c|}{ Tertiary lateral shoots } \\
\hline & & Number $\pm S D$ & Weight $(\mathrm{g}) \pm \mathrm{SD}$ & Number \pm SD & Weight $(\mathrm{g}) \pm \mathrm{SD}$ & Number \pm SD & Weight $(\mathrm{g}) \pm \mathrm{SD}$ \\
\hline \multirow[t]{4}{*}{1} & SAS-Ls-1 & $20.60 \pm 1.65$ & $36.79 \pm 15.34$ & $5.60 \pm 1.78$ & $9.99 \pm 5.09$ & $3.10 \pm 0.99$ & $4.27 \pm 2.46$ \\
\hline & SAS- $/ s-1$ & $23.10 \pm 1.37$ & $18.42 \pm 7.09$ & $0.00 \pm 0.00 * *$ & $0.00 \pm 0.00 * *$ & $0.00 \pm 0.00 * \star$ & $0.00 \pm 0.00 * *$ \\
\hline & SAS-Ls-2 & $20.20 \pm 2.30$ & $37.44 \pm 18.29$ & $8.40 \pm 2.55$ & $22.33 \pm 9.33$ & $1.90 \pm 1.10$ & $3.71 \pm 3.21$ \\
\hline & SAS-Is-2 & $22.56 \pm 1.33$ & $32.03 \pm 11.72$ & $0.33 \pm 0.71 * \star$ & $0.50 \pm 1.10 * *$ & $0.00 \pm 0.00 * *$ & $0.00 \pm 0.00 * *$ \\
\hline \multirow[t]{4}{*}{2} & SAS- $B / 1-1$ & $21.10 \pm 1.22$ & $57.38 \pm N C$ & $16.00 \pm 3.61$ & $64.98 \pm N C$ & $2.30 \pm 1.75$ & $17.67 \pm \mathrm{NC}$ \\
\hline & SAS-b/1-1 & $18.80 \pm 0.80$ & $42.87 \pm N C$ & $1.46 \pm 2.18$ ** & $8.67 \pm N C$ & $0.00 \pm 0.00 * \star$ & $0.00 \pm \mathrm{NC}$ \\
\hline & SAS- $B / 1-2$ & $22.00 \pm 1.71$ & $155.01 \pm N C$ & $19.83 \pm 2.86$ & $56.13 \pm N C$ & $12.17 \pm 5.86$ & $57.02 \pm \mathrm{NC}$ \\
\hline & SAS-b/1-2 & $23.60 \pm 2.28$ & $95.94 \pm N C$ & $8.36 \pm 3.82^{\star \star}$ & $49.57 \pm N C$ & $1.43 \pm 1.70 * \star$ & $5.38 \pm \mathrm{NC}$ \\
\hline \multirow[t]{2}{*}{3} & SAS-REV & $19.89 \pm 0.60$ & $85.11 \pm 39.78$ & $8.78 \pm 2.91$ & $35.63 \pm 12.48$ & $5.33 \pm 2.96$ & $17.10 \pm 14.11$ \\
\hline & SAS-rev & $19.80 \pm 0.63$ & $78.42 \pm 29.98$ & $0.00 \pm 0.00 * \star$ & $0.00 \pm 0.00 * *$ & $0.00 \pm 0.00 * \star$ & $0.00 \pm 0.00 * *$ \\
\hline
\end{tabular}

**: Significantly different from the wild type line(s) in the same trial at the $1 \%$ level by $t$-test.

NM: Not measured

NC: Not calculable

Table 5. Agronomic characteristics in field trials at commercial production sites 


\begin{tabular}{|c|c|c|c|c|c|c|c|c|c|c|c|c|c|c|}
\hline \multirow[t]{2}{*}{ Location } & \multirow[t]{2}{*}{ Line } & \multirow{2}{*}{$\begin{array}{l}\text { Days } \\
\text { to } \\
\text { flower }\end{array}$} & \multirow{2}{*}{$\begin{array}{l}\text { Plant } \\
\text { height } \\
\text { (cm) }\end{array}$} & \multirow{2}{*}{$\begin{array}{l}\text { Number } \\
\text { of } \\
\text { leaves }\end{array}$} & \multicolumn{3}{|c|}{ Leaf at $1 / 4$ from the top } & \multicolumn{3}{|c|}{ Leaf at $1 / 2$ from the top } & \multicolumn{3}{|c|}{ Leaf at $3 / 4$ from the top } & \multirow{2}{*}{$\begin{array}{l}\text { Lea } \\
\text { yiel } \\
\text { (kg/ } / \\
\text { a) }\end{array}$} \\
\hline & & & & & $\begin{array}{l}\text { Length } \\
\text { (cm) }\end{array}$ & $\begin{array}{l}\text { Width } \\
(\mathrm{cm})\end{array}$ & $\begin{array}{l}\text { Color } \\
\text { scale }^{1}\end{array}$ & $\begin{array}{l}\text { Length } \\
\text { (cm) }\end{array}$ & $\begin{array}{l}\text { Width } \\
(\mathrm{cm})\end{array}$ & $\begin{array}{l}\text { Color } \\
\text { scale }^{1}\end{array}$ & $\begin{array}{l}\text { Length } \\
\text { (cm) }\end{array}$ & $\begin{array}{l}\text { Width } \\
(\mathrm{cm})\end{array}$ & $\begin{array}{l}\text { Color } \\
\text { scale }^{1}\end{array}$ & \\
\hline \multirow[t]{2}{*}{1} & Coker319 & 69.3 & 128.0 & 19.9 & 43.5 & 12.0 & 8.2 & 56.0 & 20.5 & 6.4 & 56.1 & 27.5 & 5.8 & 281 \\
\hline & Coker319-/s-1 & $69.9 *$ & $134.1^{\star \star}$ & 20.2 & 45.2 & 14.0 ** & 8.2 & 57.1 & $23.3^{* *}$ & $6.9 * *$ & 56.6 & $30.3^{* *}$ & 6.0 & 305 \\
\hline \multirow[t]{2}{*}{2} & Coker319 & 72.3 & 110.5 & 22.1 & 48.7 & 13.1 & 7.5 & 66.6 & 20.9 & 7.4 & 59.8 & 28.6 & 7.0 & 191 \\
\hline & Coker319-/s-1 & $73.5^{\star}$ & $115.0^{*}$ & 22.5 & 49.9 & 13.8 & 7.2 & 65.8 & $23.4^{\star \star}$ & 7.1 & $56.2^{\star \star}$ & 28.8 & 6.9 & 253 \\
\hline \multirow[t]{2}{*}{3} & Coker319 & 69.1 & 132.5 & 19.4 & 44.8 & 10.9 & 8.0 & 55.5 & 19.0 & 7.1 & 58.3 & 27.1 & 6.3 & 268 \\
\hline & Coker319-/s-1 & $69.9 *$ & 133.0 & 19.6 & $48.5^{\star \star}$ & $13.3^{* \star}$ & 8.0 & $59.2^{\star \star}$ & $23.3^{* *}$ & $7.7^{\star \star}$ & 59.5 & $30.1^{\star *}$ & $6.8^{* *}$ & 271 \\
\hline \multirow[t]{2}{*}{4} & Coker319 & 59.6 & 134.3 & 17.1 & 60.0 & 18.3 & 8.9 & 69.7 & 25.0 & 8.8 & 65.4 & 29.2 & 8.0 & 236 \\
\hline & Coker319-/s-1 & 61.0 ** & 131.6 & $18.3^{*}$ & $63.4^{*}$ & 22.9 ** & 9.0 & 72.2 & $29.4^{\star *}$ & 8.9 & 62.4 & 29.3 & 8.2 & 267 \\
\hline \multirow[t]{2}{*}{5} & Coker319 & 63.3 & 129.8 & 19.1 & 50.8 & 14.0 & 8.4 & 62.5 & 24.9 & 7.8 & 58.4 & 27.8 & 6.5 & 218 \\
\hline & Coker319-b/1-1 & 63.9 & $119.5^{\star \star}$ & $20.7 \star \star$ & 50.1 & 13.4 & 8.7 & 61.8 & $23.1^{*}$ & 7.9 & 52.2 & 27.2 & 6.6 & 218 \\
\hline \multirow[t]{2}{*}{6} & Coker319 & 65.7 & 114.3 & 22.4 & 37.1 & 11.5 & 7.5 & 52.8 & 18.6 & 7.7 & 56.3 & 27.3 & 6.4 & 293 \\
\hline & Coker319-b/1-1 & 66.2 & 116.3 & $24.7 \star \star$ & 35.1 & 11.1 & 7.4 & 54.0 & 17.3 & 7.5 & 57.3 & 28.5 & $6.1^{*}$ & 288 \\
\hline
\end{tabular}

*, **: Significantly different from wild type Coker319 at the same location at the $5 \%$ level and the $1 \%$ level, respectively, by the $t$-test

${ }^{1}$ Larger numbers are shown with the darker colors.

2 Yield of cured leaves

\section{Figures}

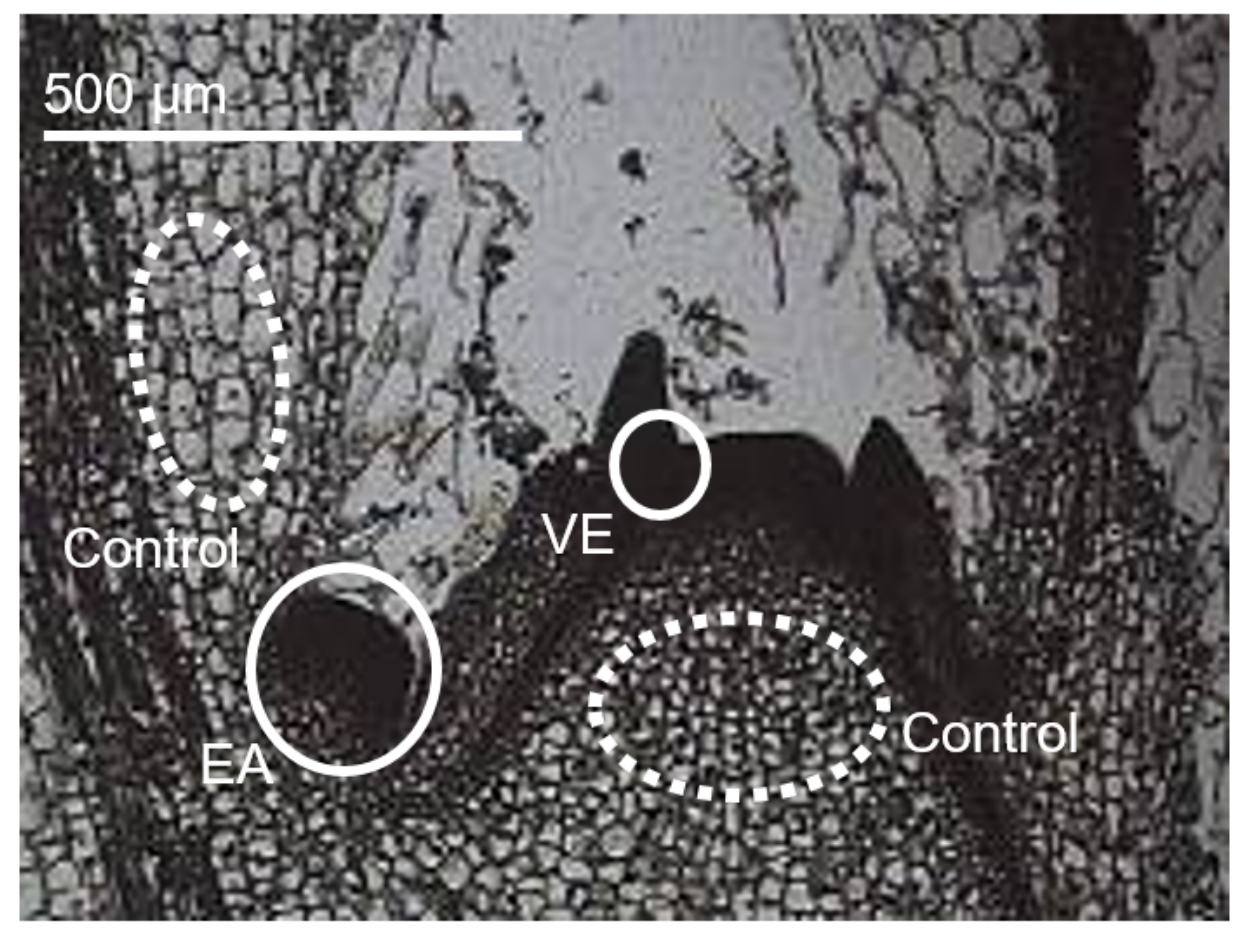

\section{Figure 1}

Tissues of early (EA) and very early (VE) stages of axillary meristems and control tissues sampled by laser micro-dissection. 

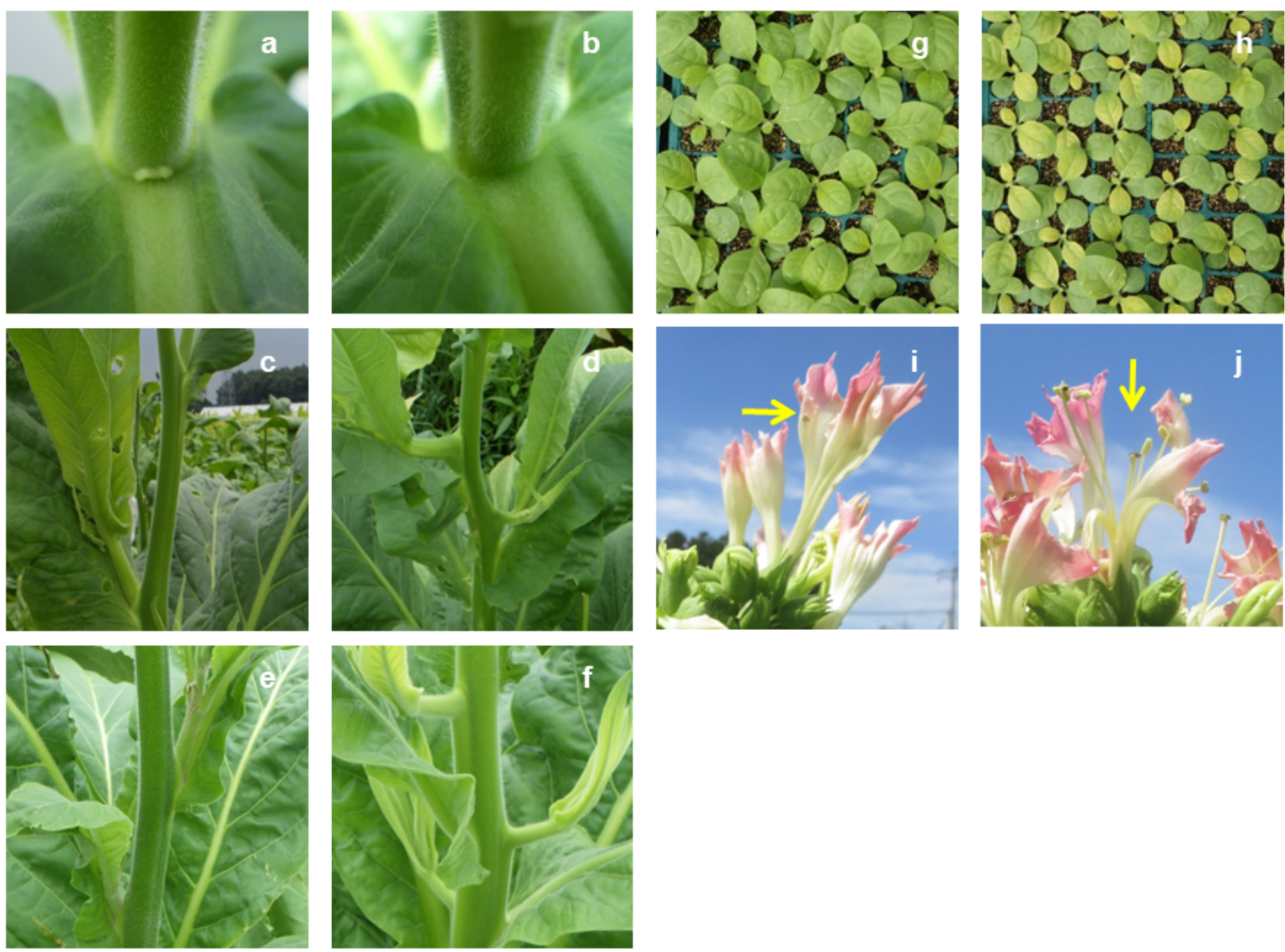

Figure 2

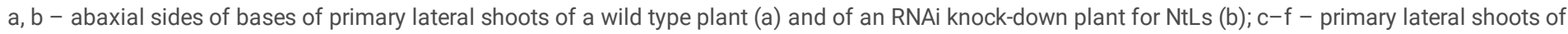
wild type plants (c, e), SAS-ls-1 (d), and SAS-bl1-1 (f); g, h - seedlings of wild type (g) and Coker319-ls-1 (h); i, j - flowers of Coker319-ls-1.

(a) NtLs

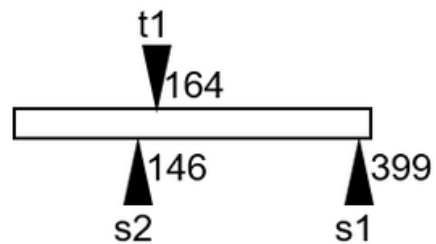

(c) NtREV (b) NtB/1

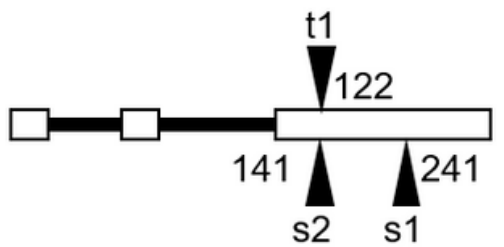

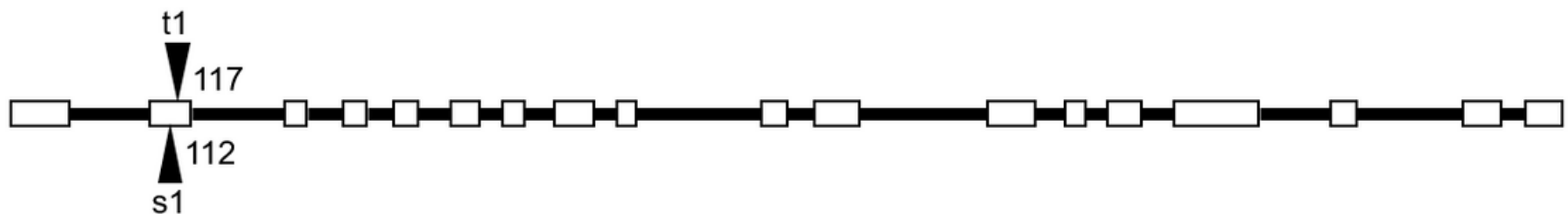

Figure 3

Locations of chemically induced loss-of-function mutations. Numbers show positions of the nonsense codons from the start of the open reading frame. Exons are represented by open boxes and introns by black lines. 


\section{Supplementary Files}

This is a list of supplementary files associated with this preprint. Click to download.

- SupplementalFig.S1.pptx

- SupplemetalFig.S2.pptx

- SupplementalTables.docx 